\title{
Comparison of Postoperative Outcomes Between Laparoscopic and Open Surgery for Colorectal Cancer
}

\author{
KAI NEKI, KEN ETO, MAKOTO KOSUGE, MASAHISA OHKUMA, ROTA NOAKI, \\ RYOSUKE HASHIZUME, SHIGEMASA SASAKI, YOSHIHIRO SHIRAI and KATSUHIKO YANAGA \\ Department of Surgery, The Jikei University School of Medicine, Tokyo, Japan
}

\begin{abstract}
Background/Aim: To determine the superiority of the laparoscopic vs. open technique for colorectal cancer surgery. Patients and Methods: We performed a retrospective analysis of consecutive patients who underwent curative surgery by laparoscopic colectomy (LC) or open colectomy $(O C)$ for colon cancer. The patients were classified into two groups: as LC group and OC group. We retrospectively assessed clinical characteristics, intraoperative and postoperative outcomes and long-term outcomes between the two groups by univariate analysis. Results: The LC group had significantly less intraoperative blood loss, complications, and shorter post-operative hospital stay than the OC group. The overall survival of Stage II in the LC group is significantly longer than the OC group. DFS of Stage III in the LC group was significantly longer than the OC group. Conclusion: LC showed more favorable results in both short-term and long-term outcomes than OC.
\end{abstract}

The incidence and mortality rate of colorectal cancer (CRC) have been increasing. CRC is the fourth and third most common cancer of the world in men and women, respectively. In addition, CRC is the second leading cause of cancer-related death in many developed countries (1). The mortality rate of colorectal cancer has been decreasing by 2 $3 \%$ annually (2) due to the improvements of treatment. Despite improvements of chemotherapy and radiotherapy, the only curative treatment remains appropriate surgical resection of the primary tumor. Open surgery used to be the only option available but laparoscopic resection has developed as an alternative. The laparoscopic surgery for CRC is rapidly increasing after the first report in 1991 (3).

Correspondence to: Prof. Kai Neki, Department of Surgery, The Jikei University School of Medicine, 3-25-8, Nishi-shinbashi, Minato-ku, Tokyo, 105-8461, Japan. Tel: +81 334331111 ext. 3401, Fax: +81354724140, e-mail: k-neki.05@jikei.ac.jp

Key Words: Laparoscopic colorectal surgery, short-term outcome, long-term outcome.
As for laparoscopic surgery, it is one of the modern surgical techniquesusing small incisions (usually $0.5-1.5 \mathrm{~cm}$ long), compared to the larger incisions (usually $24-36 \mathrm{~cm}$ long) needed in traditional open surgery (4). A number of available prospective randomized control trials and meta-analyses of laparoscopic-assisted surgery for CRC (5-10) reported the superiority of laparoscopic-assisted colorectal surgery over open surgery in terms of improved perioperative outcomes including less pain, a smaller incision, a faster recovery of gastrointestinal function, a shorter post-operative hospital stay, while long-term survival were comparable between the two groups (11-14). Laparoscopic surgery, which utilizes a telescopic video camera, usually gives a better view of internal organs than that with the naked eyes by traditional open procedures. Due to more advantages, laparoscopic surgery for CRC is now widely conducted in many facilities. The present study aimed to compare the clinical outcomes of laparoscopic-assisted surgery versus open surgery for CRC and investigate the oncological safety and potential advantages and disadvantages of laparoscopic-assisted surgery for CRC.

\section{Patients and Methods}

The subjects were 546 patients who underwent CRC surgery during the period from January 2008 to June 2011 at the Department of Surgery, the Jikei University Hospital, Tokyo, Japan. This study was approved by the institutional review board (27-2838168).

The patients were classified into two groups as laparoscopic colectomy (LC group; $\mathrm{n}=254$ ) and open colectomy (OC group; $\mathrm{n}=292$ ). Prior to surgery, the patients underwent image studies such as colonoscopy, computed tomography (CT) and/or magnetic resonance image (MRI) and procedures were decided at conferences within our hospital. Thereafter, the patients were allocated to laparoscopic or conventional open surgery groups based on the attending surgeons' discretion.

We retrospectively compared clinical characteristics, intraoperative and postoperative outcomes and disease-free survival (DFS), as well as overall survival (OS) between two groups by univariate analysis. The factors consisted of the following factors; age, gender, body mass index (BMI), American Society of Anesthesiologists (ASA) score, tumor location, concomitant 
Table I. Clinical characteristics of patients.

\begin{tabular}{lccc}
\hline & LC $(\mathrm{n}=254)$ & OC (n=292) & $p$-Value \\
\hline Age (years) & $65.0 \pm 10.6$ & $66.4 \pm 11.0$ & 0.133 \\
Gender (M:F) & $165: 89$ & $185: 107$ & 0.697 \\
BMI ( $\geq 25:<25)$ & $80: 174$ & $81: 211$ & 0.337 \\
ASA (1:2 or 3) & $89: 165$ & $101: 191$ & 0.928 \\
Rectum: Colon & $96: 158$ & $95: 197$ & 0.209 \\
Concomitant procedure (Yes:No) & $9: 245$ & $42: 250$ & 0.001 \\
Emergency surgery (Yes:No) & $253: 1$ & $277: 15$ & 0.001 \\
Stage (0:I:II:III:IV) & $24: 129: 40: 48: 13$ & $6: 30: 80: 105: 71$ & 0.001 \\
\hline
\end{tabular}

LC: Laparocopic colectomy; OC: open colectomy.

Table II. Intraoperative data and post-operative outcomes.

\begin{tabular}{lccc}
\hline & LC $(\mathrm{n}=254)$ & OC $(\mathrm{n}=292)$ & $\mathrm{p}$-Value \\
\hline Time (min) & $241.7 \pm 79.0$ & $198.6 \pm 97.2$ & $<0.001$ \\
Blood loss (ml) & $52.7 \pm 161.6$ & $391.9 \pm 538.2$ & $<0.001$ \\
CD 1 or 2 complications & \multicolumn{2}{c}{} & $<0.001$ \\
$\quad$ Yes & 17 & 56 & \\
$\quad$ No & 237 & 236 & 0.024 \\
CD 3 or 4 complications & 10 & 266 & $<0.001$ \\
$\quad$ Yes & 244 & $19.4 \pm 13.8$ & \\
$\quad$ No & $11.5 \pm 7.4$ & & \\
Post-operative hospital stays (days) & & & \\
\hline
\end{tabular}

LC: Laparocopic colectomy; OC: open colectomy.

procedures, emergency or elective surgery, tumor stage, operation time, estimated intraoperative blood loss, postoperative complications (Clavien-Dindo classification 1 or 2 and 3 or 4) (1517) and postoperative hospital stay. Recurrence of CRC was defined as newly detected local or distant metastatic tumors by CT or MRI with or without increase in serum carcinoembryonic antigen (CEA) or carbohydrate antigen 19-9 (CA19-9). All excised specimens were diagnosed at the Department of Pathology, the Jikei University Hospital. The pathological diagnosis was based on Japanese classification of CRC edition X (18).

Univariate analysis was performed using the Student's t-test and the Peason's Chi-square test. Analysis of DFS and OS were performed using the log-rank test, and the survival curve was calculated using the Kaplan-Meier estimator. All $p$-values were considered statistically significant when the associated probability was less than 0.05 .

\section{Results}

Patients' characteristics are summarized in Table I. There were no significant differences in age, gender, BMI, ASA score and type of surgical procedure between the two groups. The OC group had more cases of colectomy with concomitant procedures $(p<0.001)$. Emergency surgeries were less common in the LC group than the OC group $(p<0.001)$.
Perioperative outcomes are summarized in Table II. Operation time was shorter in the OC group than the LC group $(p<0.001)$. The LC group had significantly fewer CD 1 or 2 and CD 3 or 4 complications than the OC group ( $p<0.001$ and $p=0.024$ respectively). The mean postoperative hospital stay in the LC group was 11.5 days, which was 7.9 days shorter than the OC group $(p<0.001)$.

Long-term outcomes are shown in Figures 1 and 2. In Stage 0 , I and IV, there were no significant differences between 2 groups in OS and DFS (Figure 1A, B, E and Figure 2A). In Stage II, the OS in the OC group was significantly shorter than the LC group ( $p=0.043$ ) (Figure $1 C)$, whereas, there were no significant differences between 2 groups in DFS (Figure 2B). The DFS showed significant difference only in the stage III, with the OC group significantly shorter than the LC group ( $p=0.003$ ) (Figure $2 \mathrm{C}$ ), whereas, there were no significant differences between 2 groups in OS (Figure 1D).

\section{Discussion}

Since Jacobs et al. (14) completed the first laparoscopicassisted colectomy in 1991, laparoscopy has been an integral 

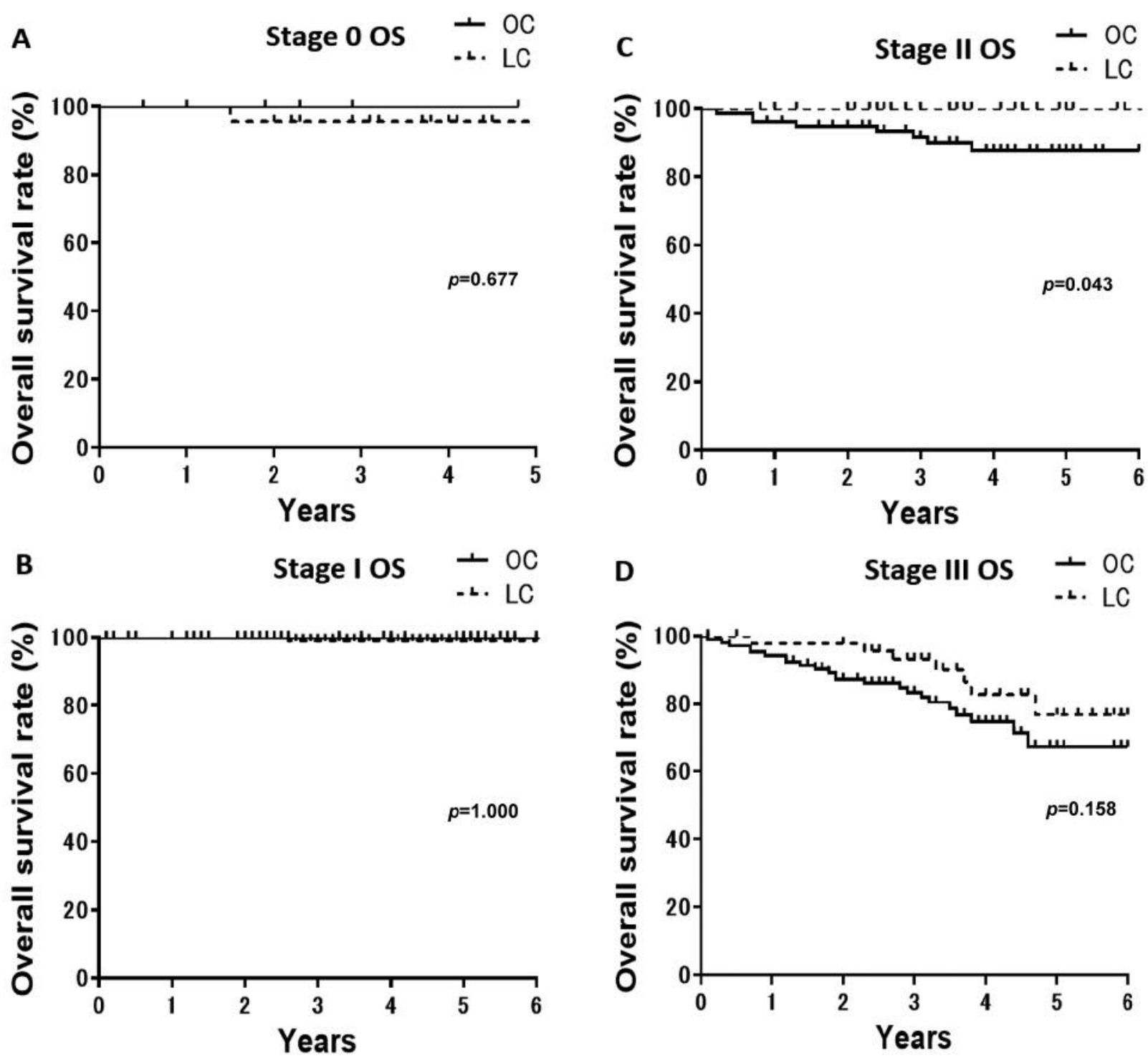

Figure 1. Overall survival rate of $L C$ versus $O C$ group. (A) Stage 0. (B) Stage I. (C) Stage II. (D) Stage III. (E) Stage IV.

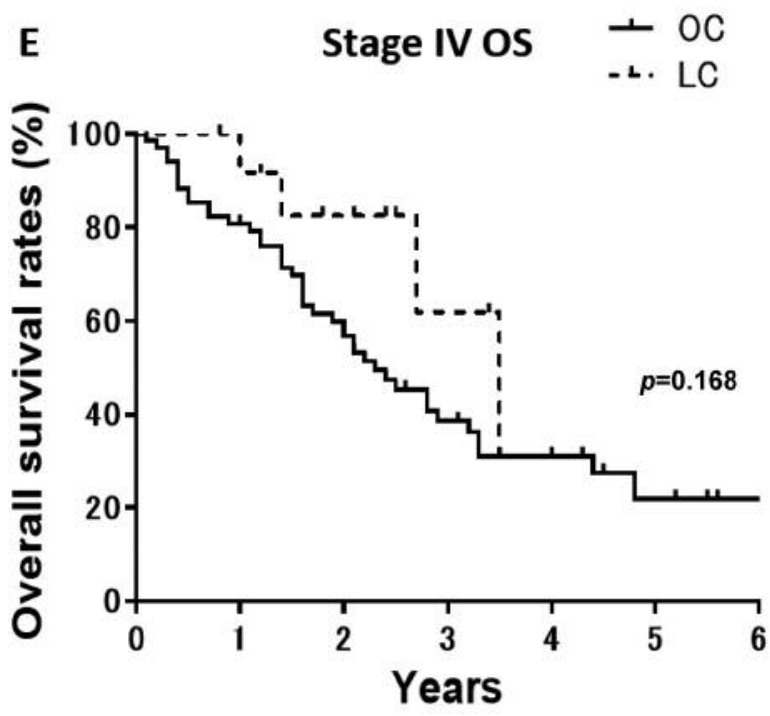


Table III. T-factor examination at stage III.

\begin{tabular}{lccc}
\hline & LC $(\mathrm{n}=43)$ & OC $(\mathrm{n}=98)$ & $p$-Value \\
\hline T1 or T2 & 8 & 3 & \\
T3 or T4 & 35 & 95 & 0.003 \\
\hline
\end{tabular}

LC: Laparocopic colectomy; OC: open colectomy.

part of the practice of general and colorectal surgery. In 2007, Fleshman et al. (19) reported that LC was not only better in short-term outcomes, but also not worse in longterm outcomes. A number of previous studies reported that patients who underwent laparoscopic surgery for CRC possessed several advantages such as less bleeding, less trauma, a faster recovery of bowel function and shorter hospital stay $(11,20,21)$.

In the current series, due to laparoscope's magnifying abilities which enable surgeons to detect small vessels, the amount of bleeding was less in the LC group than in the OC group $(p<0.001)$. In addition, CD 1 or 2 and 3 or 4 complications in the LC group was less than the OC group $(p<0.001$ and $p=0.024$ respectively), and consequently these favorable outcomes resulted in short hospital stay after surgery in LC group $(p<0.001)$. These results correlated with less blood loss $(p<0.001)$, less emergent surgery $(p<0.001)$ and less concomitant procedures $(p<0.001)$ in LC group. Generally, the complication rates of LC are reported as 11$21 \%(6,13,19)$, and results were comparable.

As to the significantly longer operative time of the LC group, this seems attributable to complicate techniques in desquamations and mobilizations. This result was consistent with the past randomized controlled trial reported by Lacy et al. in 2002 (4).

As to the longer survival of stage II CRC in the LC group, the background characteristics such as number of cases, simultaneous other operation, emergency surgery and clinical stage, seems responsible. In the future, a large number of cases or a prospective study are needed. As to the better DFS of stage III, Table III suggests that more cases of shallower tumor invasion in the LC group seem responsible. T-factors in CRC could be an independent prognostic factor for DFS (22). However, there were no significant differences in OS in the patients with stage III. This is the reason why chemotherapies for colorectal cancer are advancing dramatically in recent years. FOLFIRI and FOLFOX based on 5-FU/leucovorin combined with irinotecan and oxaliplatin, and also with molecularly targeted drugs have improved the median overall survival of the recurred colorectal cancer to over 30 months.
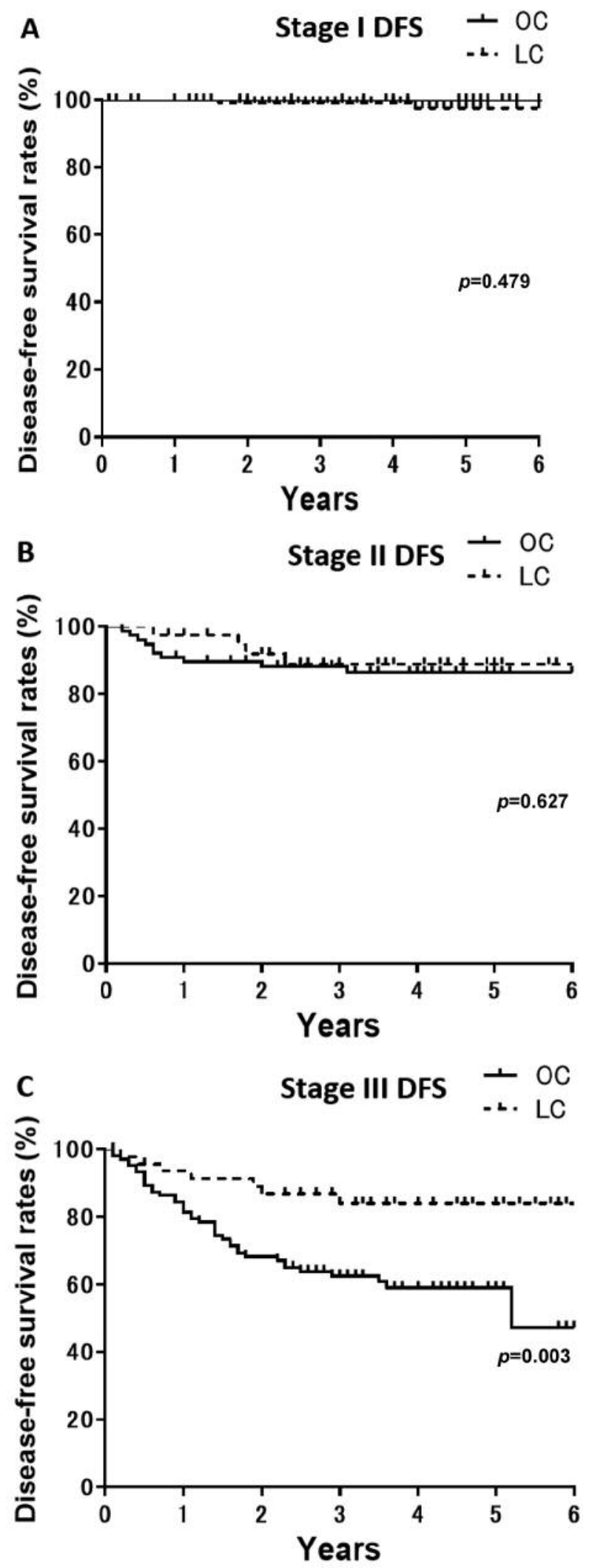

Figure 2. Disease-free survival rate of $L C$ versus OC groups. (A) Stage I. (B) Stage II. (C) Stage III. 
As discussed above, laparoscopic colectomy conducted in our department was thought to be equal or better than the open colectomy in both perioperative and long-term outcomes in the patients with colon cancer.

\section{Conclusion}

LC conducted in our hospital presented favorable results in comparison to $\mathrm{OC}$ in both short-term and long-term outcomes.

\section{Conflicts of Interest}

Authors have no conflicts of interest.

\section{References}

1 Center MM, Jemal A and Ward E: International trends in colorectal cancer incidence rates. Cancer Epidemiol Biomarkers Prev 18(6): 1688-1694, 2009.

2 Siegel R, Desantis C and Jemal A: Colorectal cancer statistics, 2014. CA Cancer J Cline 64(2): 104-117, 2014.

3 Weeks JC, Nelson H, Gelber S, Sargent D and Schroeder G; Clinical Outcomes of Surgical Therapy (COST) Study Group: Short-term quality-of-life outcomes following laparoscopicassisted colectomy $v s$. open colectomy for colon cancer: a randomized trial. JAMA 287(3): 321-328, 2002.

4 Lacy AM, García-Valdecasas JC, Delgado S, Castells A, Taurá $\mathrm{P}$, Piqué JM and Visa J: Laparoscopy-assisted colectomy versus open colectomy for treatment of non-metastatic colon cancer: a randomised trial. Lancet 359(9325): 2224-2229, 2002.

5 Abraham NS, Young JM and Solomon MJ: Meta-analysis of short-term outcomes after laparoscopic resection for colorectal cancer. Br J Surg 91(9): 1111-1124, 2004.

6 Aziz O, Constantinides V, Tekkis PP, Athanasiou T, Purkayastha S, Darzi AW and Heriot AG: Laparoscopic versus open surgery for rectal cancer: a meta-analysis. Ann Surg Oncol 31(4): 805$811,2006$.

7 Neudecker J, Klein F, Bittner R, Carus T, Stroux A and Schwenk W; LAPKON II Trialists: Short-term outcomes from a prospective randomized trial comparing laparoscopic and open surgery for colorectal cancer. Br J Surg 96(12): 1458-1467, 2009.

8 Levy BF, Tilney HS, Dowson HM and Rockall TA: A systematic review of postoperative analgesia following laparoscopic colorectal surgery. Colorectal Dis 12(1): 5-15, 2009.

9 Cancer Laparoscopic or Open Resection Study Group, Buunen M, Veldkamp R, Hop WC, Kuhry E, Jeekel J, Haglind E, Påhlman L, Cuesta MA, Msika S, Morino M, Lacy A and Bonjer HJ: Colon : Survival after laparoscopic surgery versus open surgery for colon cancer: long-term outcome of a randomised clinical trial. Lancet Oncol 10(1): 44-52, 2009.

10 Braga M, Frasson M, Zuliani W, Vignali A, Pecorelli N and Di Carlo V: Randomized clinical trial of laparoscopic versus open left colonic resection. Br J Surg 97(8): 1180-1186, 2010.

11 Biondi A, Grosso G, Mistretta A, Marventano S, Toscano C, Gruttadauria S and Basile F: Laparoscopic-assisted versus open surgery for colorectal cancer: short- and long-term outcomes comparison. J Laparoendosc Adv Surg Tech A 23(1): 1-7, 2013.
$12 \mathrm{Li}$ Z, Ying X, Shen Y, Ye P, Pan W and Chen H: Laparoscopic versus open surgery for rectal cancer: a clinical comparative study. J Int Med Res 40(4): 1599-1607, 2012.

13 Sun J, Jiang T, Qiu Z, Cen G, Cao J, Huang K, Pu Y, Liang H, Huang $\mathrm{R}$ and Chen S: Short-term and medium-term clinical outcomes of laparoscopic-assisted and open surgery for colorectal cancer: a single center retrospective case-control study. BMC Gastroenterol 27: 11-85, 2011.

14 Nelson H, Sargent D, Wieand HS et al; for the Clinical Outcomes of Surgical Therapy Study Group: Laparoscopically assisted colectomy is as safe and effective as open colectomy in people with colon cancer. Cancer Treat Rev 30(8): 707-9, 2014.

15 Clavien PA, Sanabria JR and Strasberg SM: Proposed classification of complications of surgery with examples of utility in cholecystectomy. Surgery 111(5): 518-526, 1992.

16 Dindo D, Demartines $\mathrm{N}$ and Clavien PA: Classification of surgical complications: a new proposal with evaluation in a cohort of 6336 patients and results of a survey. Ann Surg 240(2): 205-213, 2004.

17 Clavien PA, Barkun J, de Oliveira ML, Vauthey JN,Dindo D, Schulick RD, de Santibañes E, Pekolj J, Slankamenac K, Bassi C, Graf R, Vonlanthen R, Padbury R, Cameron JL and Makuuchi $\mathrm{M}$ : The Clavien-Dindo classification of surgical complications: five-year experience. Ann Surg 250(2): 187-196, 2009.

18 Watanabe T, Itabashi M, Shimada Y, Tanaka S, Ito Y, Ajioka Y, Hamaguchi $\mathrm{T}$, Hyodo I, Igarashi $\mathrm{M}$, Ishida $\mathrm{H}$, Ishihara $\mathrm{S}$, Ishiguro M, Kanemitsu Y, Kokudo N, Muro K, Ochiai A, Oguchi M, Ohkura Y, Saito Y, Sakai Y, Ueno H, Yoshino T, Boku N, Fujimori T, Koinuma N, Morita T, Nishimura G, Sakata Y, Takahashi K, Tsuruta O, Yamaguchi T, Yoshida M, Yamaguchi N, Kotake K and Sugihara K: Japanese Society for Cancer of the Colon and Rectum (JSCCR) Guidelines 2014 for treatment of colorectal cancer. Int J Clin Oncol 20(2): 207-239, 2015.

19 Jacobs M, Verdeja JC and Goldstein HS: Minimally invasive colon resection (laparoscopic colectomy). Surg Laparosc Endosc 1(3): 144-150, 1991.

20 Fleshman J, Sargent DJ, Green E, Anvari M, Stryker SJ, Beart RW Jr., Hellinger M, Flanagan R Jr, Peters W and Nelson H; Clinical Outcomes of Surgical Therapy Study Group: Laparoscopic colectomy for cancer is not inferior to open surgery based on 5-year data from the COST Study Group trial. Ann Surg 246(4): 655-662, 2007.

21 Martel G, Crawford A, Barkun JS, Boushey RP, Ramsay CR and Fergusson DA: Expert opinion on laparoscopic surgery for colorectal cancer parallels evidence from a cumulative metaanalysis of randomized controlled trials. PloS One 7(4): e35292, 2012.

22 Yabata E, Udagawa M and Okamoto H: Effect of tumor deposits on overall survival in colorectal cancer patients with regional lymph node metastases. J Rural Med 9(1): 20-26, 2014. 